\title{
Satellite Telemetry Data Transmission Immunity from the ASI and Jamming Using DSSS Optimized PN Codes in DS-CDMA Systems
}

\author{
Mahdi Sharifi ${ }^{1}$ \& Mohammad Jafar pour jalali $^{2}$ \\ ${ }^{1}$ Dept. of Electrical Engineering, Adiban Institute of Higher Education, Garmsar, Iran. \\ ${ }^{2}$ Dept. of Electrical Engineering, Adiban Institute of Higher Education, Garmsar, Iran.
}

\begin{abstract}
In the satellite telemetry command, controlling the power of the uplink signal, radiated away from the Earth to the spacecraft and downlink signal, radiated toward the Earth form the spacecraft is very important. The Uplink is transmitted at high power because of the unlimited power, while downlink should be controlled in power because of limited spacecraft power resources. This results in a lower Power Spectral Density (PSD) for the downlink and higher PSD for the uplink, which in many cases the uplink power due to be strong enough to reach the satellites at GEO would often also violate the PSD limits in the other orbits satellite telemetry signals like LEO. In addition due to long distance between the Earth and satellite, the received signals at the both terrestrial and transponder receivers are extremely week and strongly influenced by intentional interference (like jamming) or inadvertent interferences (such as the ASI). Spreading spectrum can solve the PSD problems for both directions but consumes a wide bandwidth. Fortunately, signals can be distinguished by using different spreading codes, allowing for CDMA. The Pseudo Noise (PN) spreading codes allow using many satellites or users (located in one satellite) the same frequency with overlapping signals simultaneously and also range measurement as useful ability to track the spacecraft in the space by the expanding value of the PN codes (Doppler Shift).[1] This paper specially considers to satellite telemetry data transmission immunity from the interference using Direct Sequence Spread Spectrum (DSSS) based on CDMA namely DS-CDMA (Direct Sequence-CDMA) by the unique properties correlation functions of the Gold and M-Sequence codes. Also the codes are examined in the fading channels, Reyleigh and AWGN, in terms of the BER vs. Eb/No to compare the practical results with theoretical values.
\end{abstract}

Keywords: Satellite telemetry Data, PSD, CDMA, Spread Spectrum, ASI, Jamming, DSSS, FHSS, PN Code, Maximal Length Code, Gold code, Auto-Correlation, Cross-Correlation, Reyleigh Channel, AWGN Channel.

\section{Introduction}

Code Division Multiple Access (CDMA) has a crucial role in the satellite telemetry communications systems, especially in places where the possibility of providing the proper SNR to recipients due to intentional or inadvertent interferences would be low. This paper illustrates the use of the Spread Spectrum systems in the current satellite telemetry which can be used in the Low Earth Orbit (LEO) or Geostationary Earth Orbit (GEO). It also provides a detailed specification of the well-known M-Sequence and Gold codes modulation formats implemented. Also the Auto-Correlation and Cross-Correlation functions, respectively to lock the receiver on the desire satellite signal and avoid interference of the other resources codes as two important parameters to select an optimized code to use the RF communication to achieve the high SNR at the demodulator and maximal immunity in the communication channel are considered in this paper. In other words, to choose an optimized set of the PN code from the other codes to use in a RF communication, the correlation functions values of the codes must be exactly evaluated and one of the major determining factors of an optimized PN code is to have a low cross-correlation value with other codes and high auto-correlation to enable code synchronization. [2] This paper provides a scrutiny of two well-known codes namely Gold and M-Sequence in terms of the correlation function properties to use in the DS-CDMA systems and then examines them in the Reyleigh and AWGN fading channels.

\section{Main Used Of Spread Spectrum In Satellite Communication}

Spread spectrum can be used in support of several important satellite communication applications in facing with destructive interferences.

\section{A- Adjacent Satellite Interference (ASI)}

Some satellite communication applications require very small aperture antennas such as the airborne and other mobile communications systems where the used of big antenna is very limited. Actually, Small antennas inherently have wide radiation patterns. 


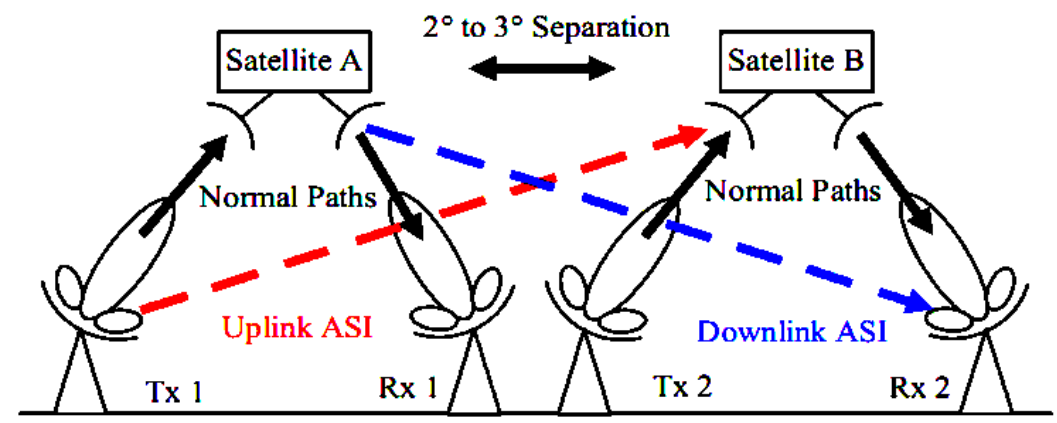

Figure.1 -Uplink and downlink ASI

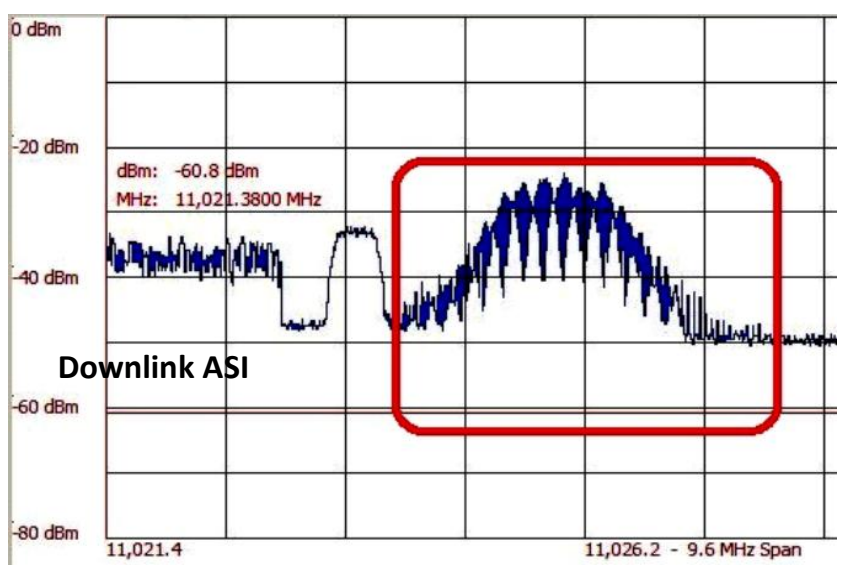

Figure.2 - Spectrum analyzer screen capturing downlink KU-Band ASI

In order to control Adjacent Satellite Inference (ASI), the FCC and other bodies governing satellite communications place some limits on the maximum PSD transmitted in the direction of an adjacent satellite by a ground based satellite terminal such as VSAT. Since Spread spectrum reduces the transmitted PSD, it can be used to enable a link budget to close thru the primary satellite while meeting PSD emission limits towards the adjacent satellite. [1]

In this case, use of spread spectrum increases the occupied bandwidth (or alternately reduces the data rate) as the key trade-off needed to enable the use of a small antenna. As shown in Figur.3, when the total transmitted power is kept constant, the PSD is reduced by a factor of N as a PN code. Since the bandwidth is increased by a factor of $\mathrm{N}$, when additive noise is added on a satellite link, the signal-to-noise ratio (SNR) of the received signal is also reduced by this same PN code. At the receiver, de-spreading the signal adds back a "processing gain" factor of $\mathrm{N}$. Hence absent any implementation loss, the net effect of spreading and despreading a signal on the overall satellite link budget is zero.

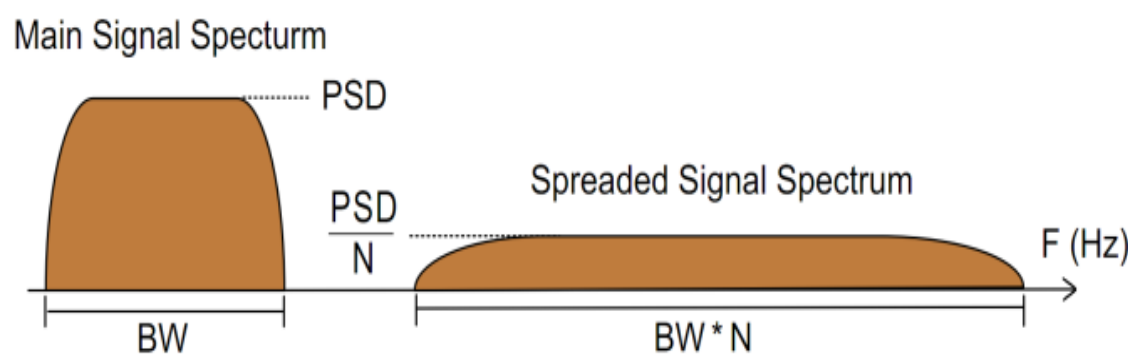

Figure.3 - Spreading signal by PN code (Factor N)

\section{B- Jamming}

Spread spectrum can be utilized to overcome jamming (either intentional or inadvertent). In the case where an interfering signal is transmitted in the same channel as a primary signal of interest, spreading can be enabled in order to maintain a connection. If the same bandwidth maintained on the satellite, the information bit rate is reduced as the key trade-off. 


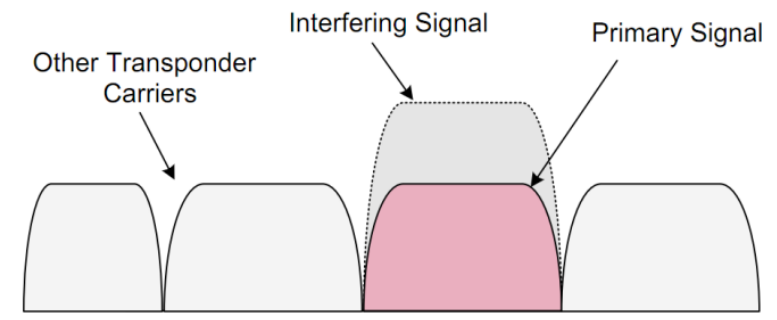

Figure.4 - Using Spread Spectrum to overcome jamming

\section{Spread Spectrum (Ss) Principles}

A transmission technique in which a pseudo-noise code (PN), independent of the information data, is employed as a modulation waveform to spread the signal energy over a much larger bandwidth than information signal bandwidth and at the receiver the signal is de-spread using a synchronized replica of the same PN code.[6] Two major types of the spread spectrum are being used in the communication systems named Direct Sequence Spread Spectrum (DSSS) and Frequency Hopping Spread Spectrum (FHSS) that only DSSS algorithms are specially focused in this paper and FHSS is just implicitly introduced.

\section{A- FHSS}

A pseudo-noise sequence PNt made in the modulator is used in connectivity with an M-Array FSK modulation to shift the carrier frequency of the FSK signal pseudo randomly, at the hopping rate (Rh). The transmitted signal occupies a number of frequencies in time, each for a period of time $\mathrm{Th}(=1 / \mathrm{Rh})$, referred to as dwell time. Actually FHSS divides the exist bandwidth into $\mathrm{N}$ channels and hops between these channels regarding the PN codes. At each frequency hop time the PN generator feeds the frequency synthesizer a frequency Word (FW) (a sequence of $n$ chips) which dictates one of $2 n$ frequency positions fhi and receiver follows the same frequency hop pattern. The transmitted bandwidth is determined by the lowest and highest hop positions and by the bandwidth per hop position $(\Delta \mathrm{fch})$. For a given hop, the instantaneous occupied bandwidth is identical to bandwidth of the conventional M-FSK, which is typically much smaller than Wss. So the FHSS signal is a narrowband signal which all transmission power is focused on the one channel. [5]

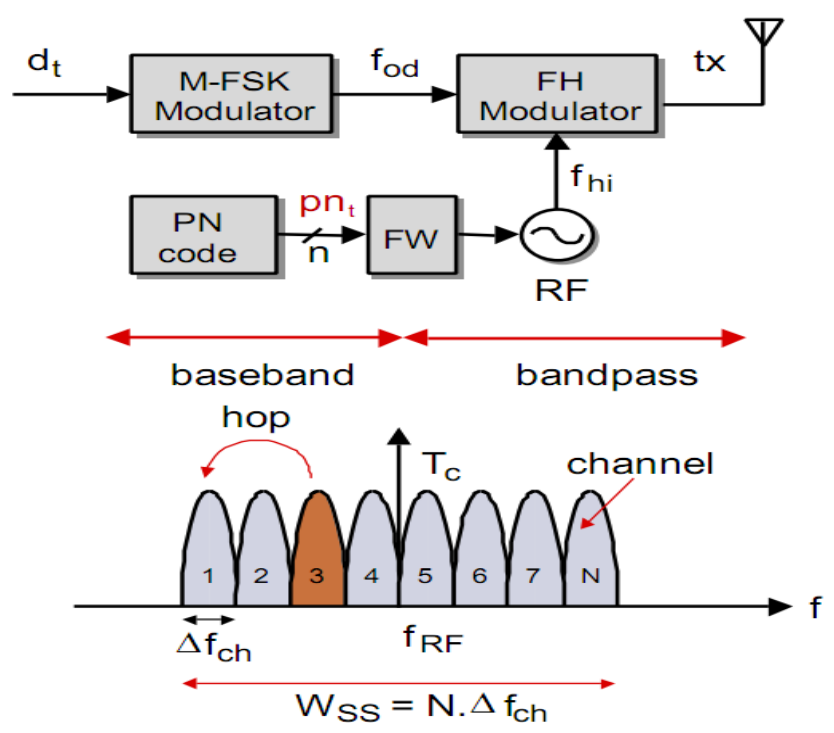

Figure.5 - FHSS / Transmitted Signal Spreading Diagram

\section{B- DSSS}

A pseudo-noise sequence pnt generated at the modulator, is used in conjunction with an M-array PSK modulation to shift the phase of the PSK signal pseudo randomly, at the chipping rate $\mathrm{Rc}(=1 / \mathrm{Tc})$ a rate that is an integer multiple of the symbol rate Rs $(=1 / \mathrm{Ts})$. The transmitted bandwidth is determined by the chip rate and by the baseband filtering. The implementation limits the maximum chip rate Rc (clock rate) and thus the maximum spreading. The PSK modulation scheme requires a coherent demodulation. A short-code system uses a PN code length equal to a data symbol. A long code system uses a PN code length that is much longer than a data symbol, so that a different chip pattern is associated with each symbol. [2] 


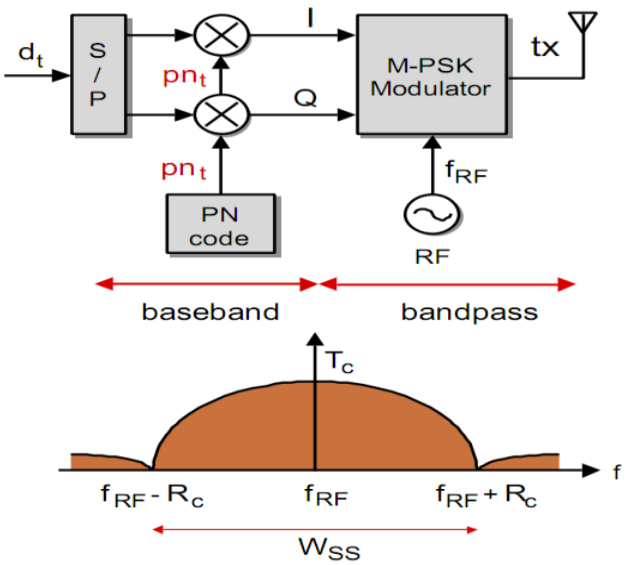

Figure.6 -Transmitted Signal Spreading Diagram in a DSSS system

The most important units in DSSS systems are the spreading part, which transmitted signal is combined with a PN code and de-spreading part, which the received signal is isolated of the PN code.

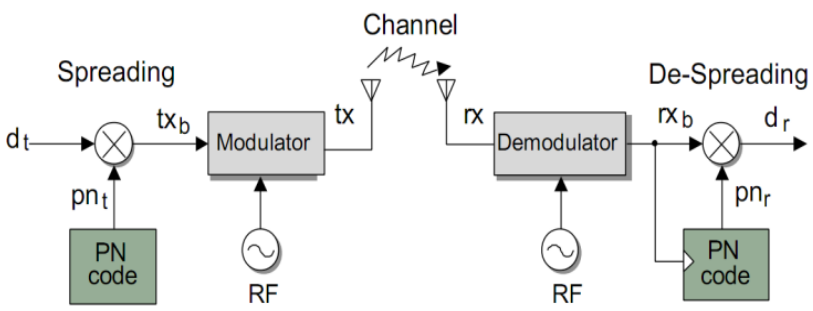

Figure.7 - DSSS system block diagram

Actually, in the transmitter, the binary data dt (for BPSK, I and Q for QPSK) is 'directly' multiplied with the PN sequence PNt, which is independent of the binary data, to produce the transmitted baseband signal txb: [5] $\mathrm{txb}=\mathrm{dt}$. PNt

The effect of multiplication of dt with a PN sequence is to spread the baseband bandwidth Rs of dt to a baseband bandwidth of Rc.

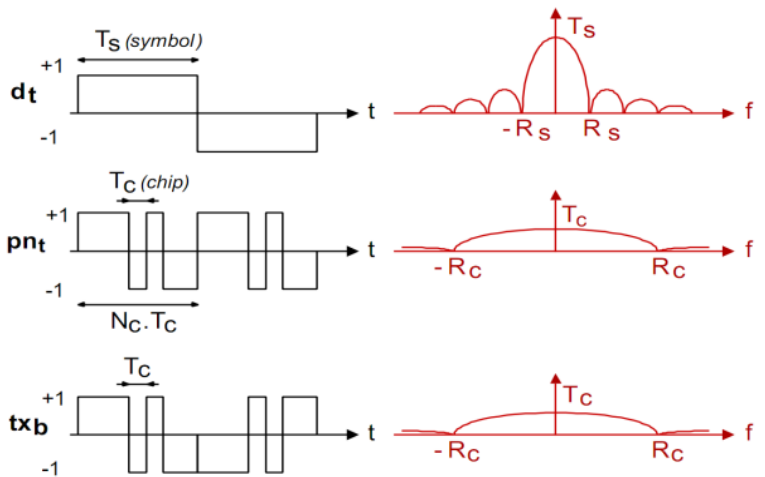

Figure.8 -Spreading signal by PNt Code

The spread spectrum signal cannot be detected by a conventional narrowband receiver. In the receiver, the received baseband signal rxb is multiplied with the PN sequence PNr. Spread Spectrum systems are spreading the information signal dt which has a BWinfo, over a much larger bandwidth BWSS: [6]

BWinfo $\approx$ Rs $\ll$ BWSS $\approx$ Rc

To de-spread the signal if $\mathrm{PNr}=\mathrm{PNt}$ and synchronized to the $\mathrm{PN}$ sequence in the received data, than the recovered binary data is produced on $\mathrm{dr}$. 


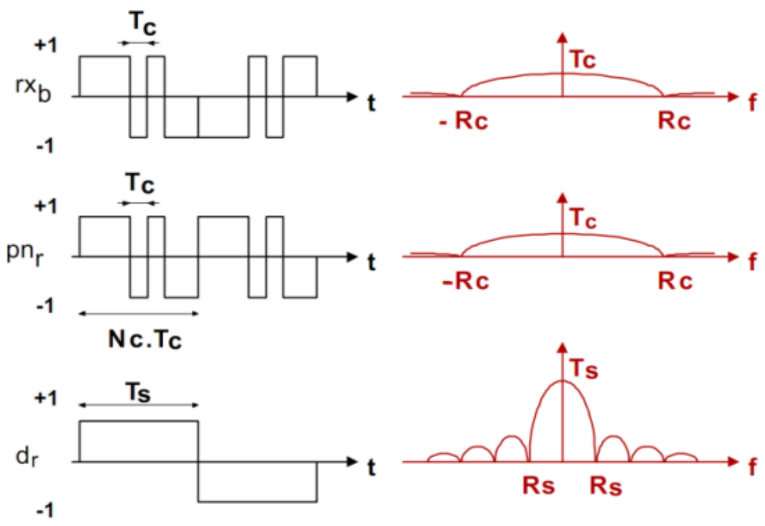

Figure.9 - De-spreading signal by $\mathrm{PNr}$ Code $(\mathrm{PNr}=\mathrm{PNt})$

The effect of multiplication of the Spread Spectrum signal rxb with the PN sequence PNt used in the transmitter is to de-spread the bandwidth of rxb to Rs but, if $\mathrm{PNr} \neq \mathrm{PNt}$, than there is no dispreading action.

The SS-signal spectrum is white noise-like. The amplitude and thus the power in the SS-signal txb is the same as in the original information signal dt. Due to the increased bandwidth of the SS signal the power spectral density must be lower. The bandwidth expansion factor, being the ratio of the chip rate Rc and the data symbol rate Rs, is usually selected to be an integer in practical SS systems: [2]

$S S=G_{p}=\frac{B W_{S S}}{B W_{\text {info }}}=\frac{R_{c}}{R_{S}}=\frac{T_{b}}{T_{c}}=N_{c}$

If the PN sequence at the receiver is not synchronized properly to the received signal, the data cannot be recovered.

\section{Immunity From Interference In Dsss Systems}

To simplify the influence of interference, the Spread Spectrum system is considered for baseband BPSK communication (without filtering).

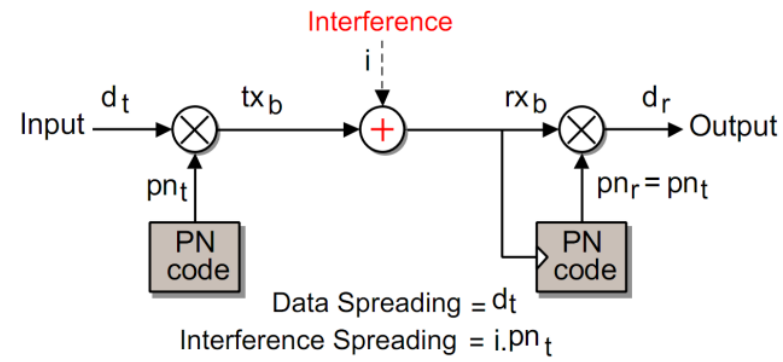

Figure.10 - DSSS system with a interfering channel

The received signal $\mathrm{rxb}$ consists of the transmitted signal txb plus an additive interference $\mathrm{i}$ (noise, other users signal, jammer, ... ):

$\mathrm{rxb}=\mathrm{txb}+\mathrm{i}=\mathrm{dt} . \mathrm{pnt}+\mathrm{i}$

To recover the original data $\mathrm{dt}$, the received signal rxb is multiplied with a locally generated PN sequence pnr that is an exact replica of that used in the transmitter (that is $\mathrm{pnr}=$ pnt and synchronized). The multiplier output is therefore given by:

$\mathrm{dr}=\mathrm{rxb} \cdot \mathrm{pnt}=\mathrm{dt} \cdot \mathrm{pnt} \cdot \mathrm{pnt}+\mathrm{i} \cdot \mathrm{pnt}$

The data signal $\mathrm{dt}$ is multiplied twice by the PN sequence pnt, whereas the unwanted interference $\mathrm{i}$ is multiplied only once. Due to the property of the PN sequnence:

pnt . pnt $=+1$ for all $\mathrm{t}$

The multiplier output becomes:

$\mathrm{dr}=\mathrm{dt}+\mathrm{i}$. pnt

The data signal $\mathrm{dt}$ is reproduced at the multiplier output in the receiver, except for the interference represented by the additive term $i$. pnt. Multiplication of the interference $i$ by the locally generated PN sequence means that the spreading code will affect the interference just as it did with the information bearing signal at the transmitter. Noise and interference, being uncorrelated with the PN sequence, become noise-like, increased in 
bandwidth and decreased in power density after the multiplier. After de-spreading, the data component dt is narrow band (Rs) whereas the interference component is wideband (Rc). By applying the dr signal to a baseband (low-pass) filter with a bandwidth just large enough to accommodate the recovery of th data signal, most of the interference component $\mathrm{i}$ is filtered out. The effect of the interference is reduced by the processing gain (Gp). [5] In any communication channel, regardless of the Additive Gaussian White Noise (AWGN), we are facing with 2 kinds of the noise or destructive interferences:

\section{A- Narrowband interference}

The narrowband noise is spread by the multiplication with the PN sequence pnr of the receiver. The power density of the noise is reduced with respect to the de-spread data signal. Only 1/Gp of the original noise power is left in the information baseband (Rs). Spreading and dispreading enables a bandwidth trade for processing gain against narrow band interfering signals. Narrowband interference would disable conventional narrowband receivers.

The essence behind the interference rejection capability of a Spread Spectrum system: the useful signal (data) gets multiplied twice by the PN sequence, but the interference signal gets multiplied only once.[3]

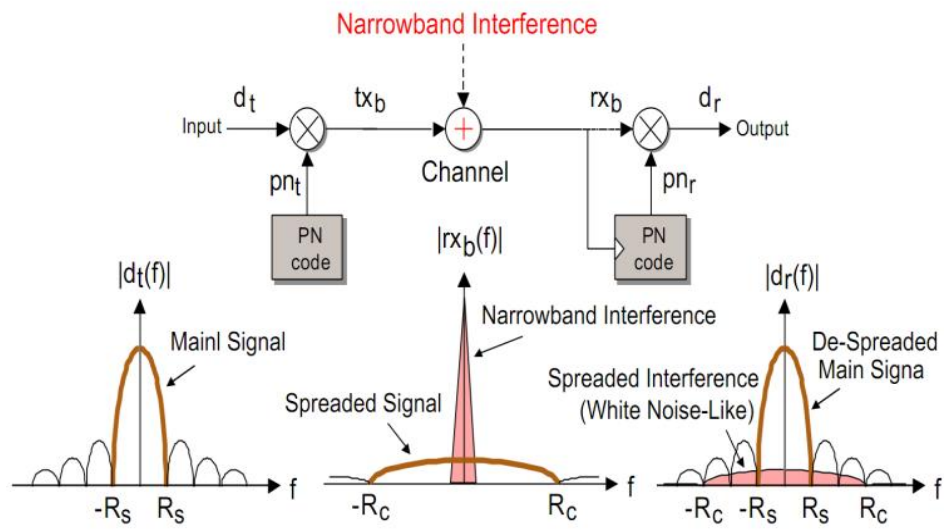

Figure.11 - DSSS performance against narrowband interference

\section{B- Wideband interference}

Multiplication of the received signal with the PN sequence of the receiver gives a selective de-spread of the data signal (smaller bandwidth, higher power density). The interference signal is uncorrelated with the PN sequence and is spread. Origin of wideband noise:

1- Multiple Spread Spectrum Users: Multiple access mechanism.

2- Gaussian Noise: There is no increase in SNR with Spread Spectrum. The larger channel bandwidth (Rc instead of Rs) increases the received noise power with Gp: [3]

Ninfo $=$ N0.BWinfo $\rightarrow$ NSS $=$ N0.BWss $=$ Ninfo.Gp

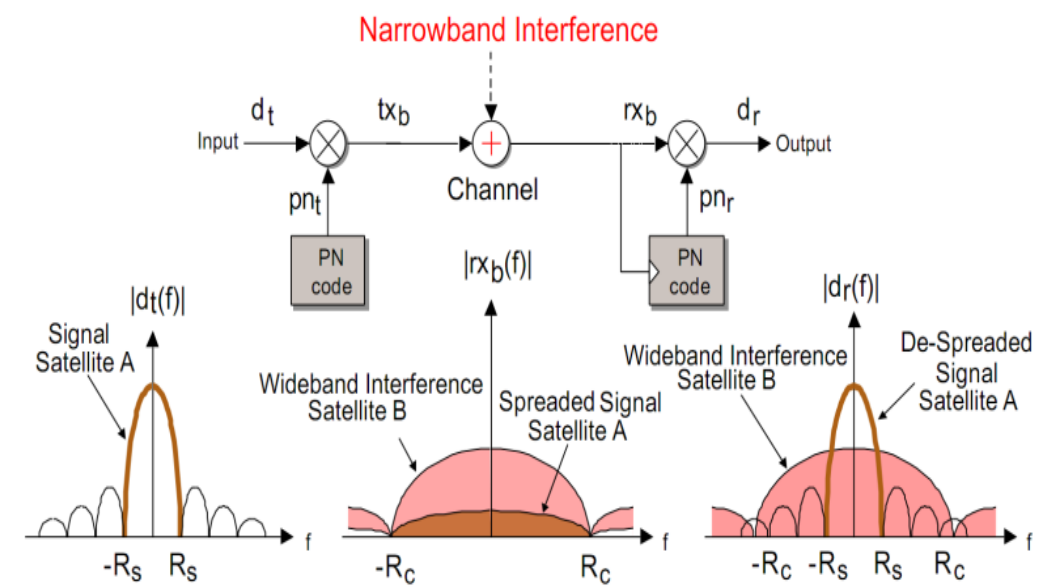

Figure.12 - DSSS performance against wideband interference 


\section{A- Rayleigh Fading Channel}

\section{Communication Channel Models}

Rayleigh fading is a statistical model for the effect of a propagation environment on a radio signal, such as that used by wireless devices. Rayleigh fading models assume that the magnitude of a signal that has passed through such a transmission medium will vary randomly, or fade, according to a Rayleigh distribution the radial component of the sum of two uncorrelated Gaussian random variables. The Bit Error Rate (BER) theoretical in this channel can be calculated by: [7]

\section{B- AWGN Channel}

$$
B E R \text { theoretical }(\text { Rayleigh })=\frac{1}{2}\left[1-\frac{1}{\sqrt{1+\frac{E_{b}}{N_{0}}}}\right]
$$

Additive White Gaussian Noise channel model as the name indicate Gaussian noise get directly added with the signal and information signal get converted into the noise in this model scattering and fading of the information is not considered. The BER theoretical in this channel can be calculated by: [7]

$$
B E R \text { theoretical }(A W G N)=\frac{1}{2} \operatorname{erfc}\left(\sqrt{E_{b} / N_{0}}\right)
$$

\section{Pseudo Noise (PN) Code Properties}

In spread-spectrum systems, the spreading signal is formed by continually repeating a finite-length PN code. To be usable for direct sequence spreading, this PN code (composed of binary units called chips to distinguish them from data bits or code symbols) should exhibit the following characteristics:

1- Codes must have a sharp autocorrelation peak to enable code synchronization.

2- The codes must have a low cross correlation value. The lower this cross-correlation, the more users can be allowed in the system. This holds for both full-code correlation and partial-code correlation. (See discussion of cross-correlation below.)

3- The codes should be 'balanced', meaning that the difference between the number of 'ones' and 'zeros' in the code may be no greater than 1 . This requirement is necessary (but not sufficient) for good spectraldensity properties (spreading the energy equally over the whole frequency band).

To choose the optimized code to use in DSSS systems the correlation functions properties of each code should be exactly evaluated and examined. [1]

\section{A- Auto-Correlation Function}

The origin of the name pseudo-noise is that the digital signal has an autocorrelation function which is very similar to that of a white noise signal: impulse like. The autocorrelation function for the periodic sequence $\mathrm{PN}$ is defined as the number of agreements less the number of disagreements in a term by term comparison of one full period of the sequence with a cyclic shift (position $\tau$ ) of the sequence itself: [2]

$$
\operatorname{Ra}(\tau)=\int_{-N c T c / 2}^{\mathrm{NcT} / 2} p n(t) \cdot p n(t+\tau) d t
$$

It is best if $\operatorname{Ra}(\tau)$ is not larger than one count if not synchronized $(\tau=0)$.

$$
\begin{aligned}
& \operatorname{pn}(0)=+1+1+1-1+1-1-1 \\
& \frac{\mathrm{pn}(0)=+1+1+1-1+1-1-1}{+1+1+1+1+1+1+1}=\Sigma=7=\operatorname{Ra}(\tau=0) \\
& \operatorname{pn}(0)=+1+1+1-1+1-1-1
\end{aligned}
$$

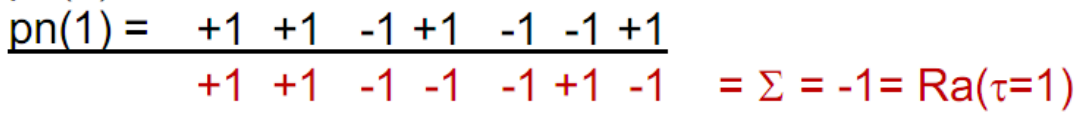




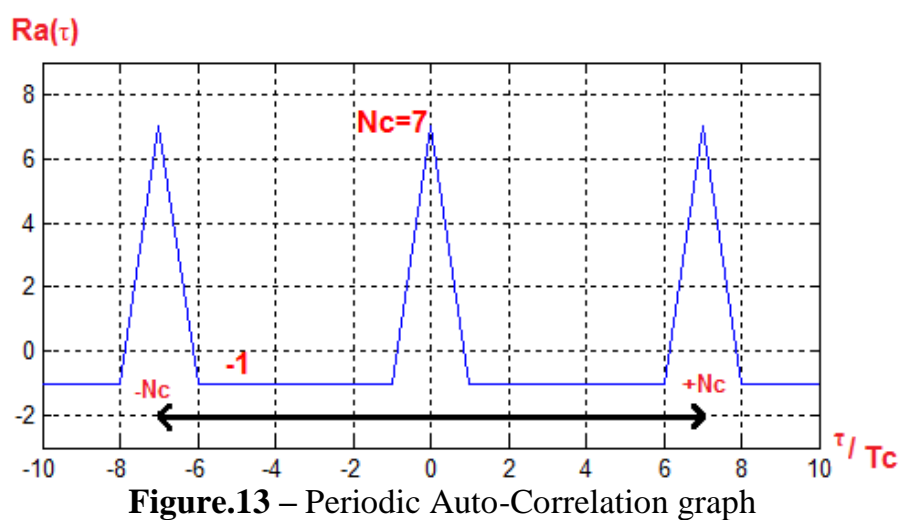

Due to the periodic nature of the PN sequence the frequency spectrum has spectral lines which become closer to each other with increasing sequence length Nc. Each line is further smeared by data scrambling, which spreads each spectral line and further fills in between the lines to make the spectrum more nearly continuous.

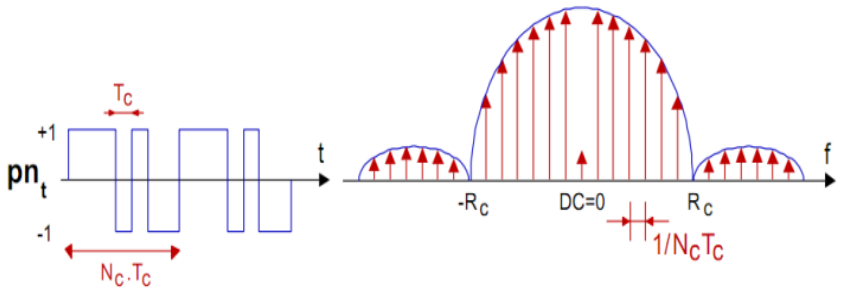

Figure.14 - Auto-Correlation in time domain and frequency domain

The DC component is determined by the zero-one balance of the PN sequence.

\section{B- Cross-Correlation Function}

Cross-correlation is the measure of agreement between two different codes $\mathrm{PNi}$ and $\mathrm{PNj}$. When the cross-correlation $\operatorname{Rc}(\tau)$ is zero for all $\tau$, the codes are called orthogonal. In CDMA multiple users occupy the same RF bandwidth and transmit simultaneous. When the user codes are orthogonal, there is no interference between the users after de-spreading and the privacy of the communication of each user is protected. In practice, the codes are not perfectly orthogonal; hence the cross correlation between user codes introduces performance degradation (increased noise power after de-spreading), which limits the maximum number of simultaneous users. Cross-correlation describes the interference between codes PNi and PNj: [2]

$$
\operatorname{Rc}(\tau)=\int_{-N_{C T C / 2}}^{\mathrm{NcTC} / 2} \mathrm{pn}_{\mathrm{i}}(\mathrm{t}) \cdot \mathrm{pn}_{\mathrm{j}}(\mathrm{t}+\tau) \mathrm{dt}
$$

\section{Close Scrutiny of DSSS Codes Correlation Values}

\section{A- M-Sequence Correlation Functions}

M-sequences are the basics of PN sequences and they are used in real systems like GPS. A Simple Shift Register Generator (SSRG) has all the feedback signals returned to a single input of a shift register (delay line). The SSRG is linear if the feedback function can be expressed as a modulo-2 sum (xor). [3]

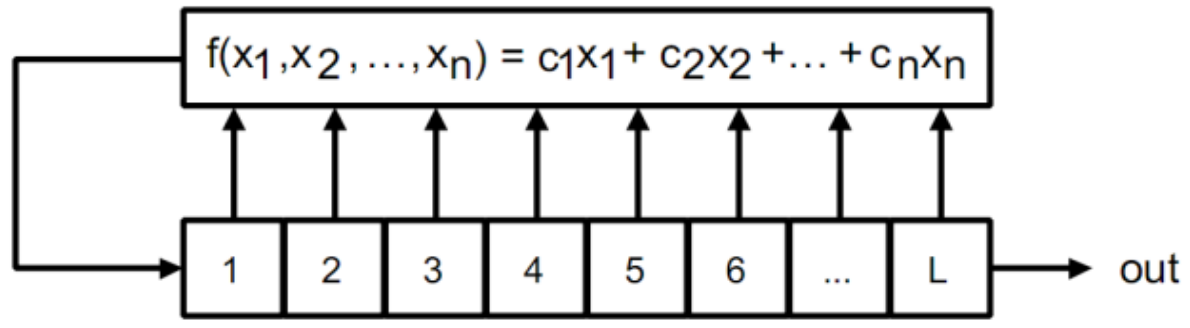

Figure.15 - Simple Shift Register Generator (SSRG) block diagram 
Satellite Telemetry Data Transmission Immunity from the ASI and Jamming Using DSSS Optimized ..

The feedback function $\mathrm{f}(\mathrm{x} 1, \mathrm{x} 2, \ldots, \mathrm{xn})$ is a modulo- 2 sum of the contents $\mathrm{xi}$ of the shift register cells with ci being the feedback connection coefficients ( $\mathrm{ci}=0=$ open, ci $=1=$ connect). An SSRG with L flip- flops produces sequences that depend upon register length $\mathrm{L}$, feedback tap connections and initial conditions. When the period (length) of the sequence is exactly Nc $=2 \mathrm{~L}-1$, the PN sequence is called a maximum length sequence or simply an m-sequence. An m-sequence generated from a linear SSRG has an even number of taps. If an Lstage SSRG has feedback taps on stages $\mathrm{L}, \mathrm{k}, \mathrm{m}$ and has sequence ..., ai, ai $+1, \mathrm{ai}+2, \ldots$ than the reverse SSRG has feedback taps on L, L-k, L $m$ and sequence ..., ai +2 , ai +1, ai, ... .

\begin{tabular}{|c|c|c|c|}
\hline L & $N_{c}=2^{L}-1$ & Feedback Taps for $\mathrm{m}$-sequences & \# m-sequences \\
\hline 2 & 3 & {$[2,1]$} & 2 \\
\hline 3 & 7 & {$[3,1]$} & 2 \\
\hline 4 & 15 & {$[4,1]$} & 2 \\
\hline 5 & 31 & {$[5,3][5,4,3,2][5,4,2,1]$} & 6 \\
\hline 6 & 63 & {$[6,1][6,5,2,1][6,5,3,2]$} & 6 \\
\hline 7 & 127 & $\begin{array}{l}{[7,1][7,3][7,3,2,1][7,4,3,2]} \\
{[7,6,4,2][7,6,3,1][7,6,5,2]} \\
{[7,6,5,4,2,1][7,5,4,3,2,1]}\end{array}$ & 18 \\
\hline 8 & 255 & $\begin{array}{l}{[8,4,3,2][8,6,5,3][8,6,5,2]} \\
{[8,5,3,1][8,6,5,1][8,7,6,1]} \\
{[8,7,6,5,2,1][8,6,4,3,2,1]}\end{array}$ & 16 \\
\hline 9 & 511 & $\begin{array}{l}{[9,4][9,6,4,3][9,8,5,4][9,8,4,1]} \\
{[9,5,3,2][9,8,6,5][9,8,7,2]} \\
{[9,6,5,4,2,1][9,7,6,4,3,1]} \\
{[9,8,7,6,5,3]}\end{array}$ & 48 \\
\hline 10 & 1023 & $\begin{array}{l}{[10,3][10,8,3,2][10,4,3,1][10,8,5,1]} \\
{[10,8,5,4][10,9,4,1][10,8,4,3]} \\
{[10,5,3,2][10,5,2,1][10,9,4,2]} \\
{[10,6,5,3,2,1][10,9,8,6,3,2]} \\
{[10,9,7,6,4,1][10,7,6,4,2,1]} \\
{[10,9,8,7,6,5,4,3][10,8,7,6,5,4,3,1]}\end{array}$ & 60 \\
\hline 11 & 2047 & $\begin{array}{l}{[11,2][11,8,5,2][11,7,3,2][11,5,3,2]} \\
{[11,10,3,2][11,6,5,1][11,5,3,1]} \\
{[11,9,4,1,][11,8,6,2,][11,9,8,3]} \\
{[11,10,9,8,3,1]}\end{array}$ & 176 \\
\hline
\end{tabular}

Table.1 - Feedback connections for m-sequence generated with a linear SSRG. [2]

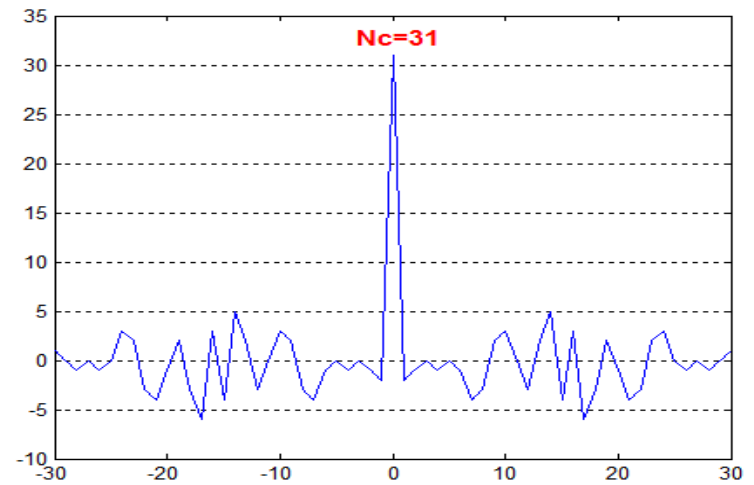

Figure.16-SSRG [5,3] $\rightarrow$ PN 1 / None periodic Auto-Correlation

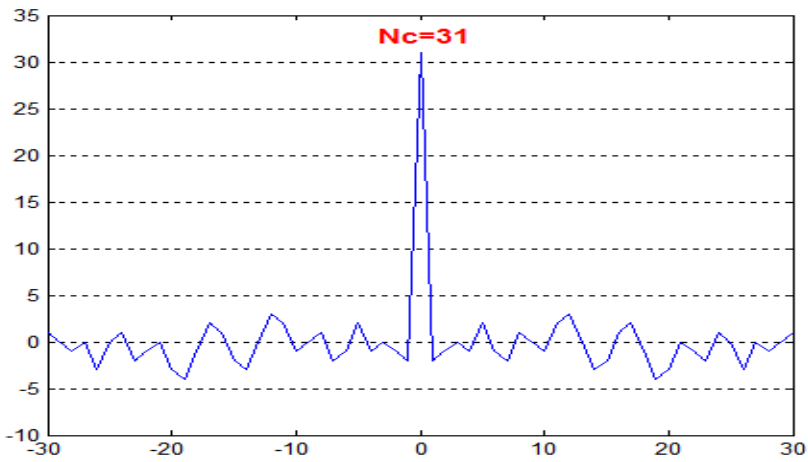

Figure.17 -SSRG [5,4,3,2] $\rightarrow$ PN 2 / None periodic Auto-Correlation 


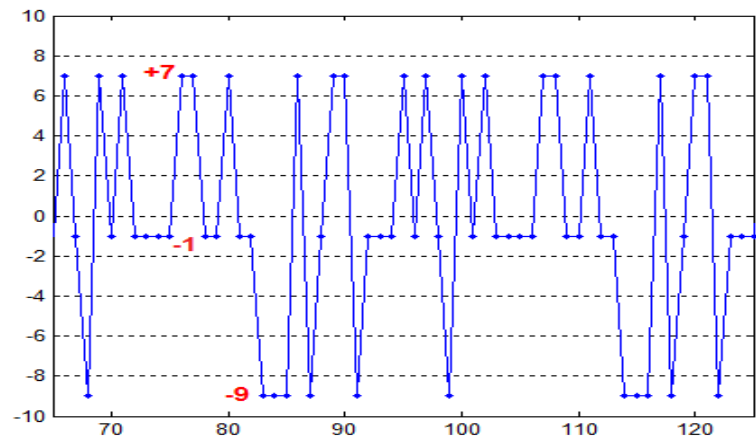

Figure.18 - PN 1 \& PN 2 / Cross-Correlation

\section{B- Gold Code Correlation Functions}

Multi-user environment system (Code Division Multiple Access) needs a set of codes with the same length and with good cross-correlation properties. Gold code sequences are useful because a large number of codes (with the same length and with controlled cross-correlation) can be generated, although they require only one 'pair' of feedback tap sets. Gold codes are product codes achieved by the exclusive (modulo-2 adding) of two maximum-length sequences with the same length (factor codes). The code sequences are added chip by chip by synchronous clocking. Because the $\mathrm{m}$ sequences are of the same length, the two code generators maintain the same phase relationship, and the codes generated are of the same length as the two base codes which are added together, but are non-maximal (so the autocorrelation function will be worse than that of m-sequences). Every change in phase position between the two generated m-sequences causes a new sequence to be generated. [5]

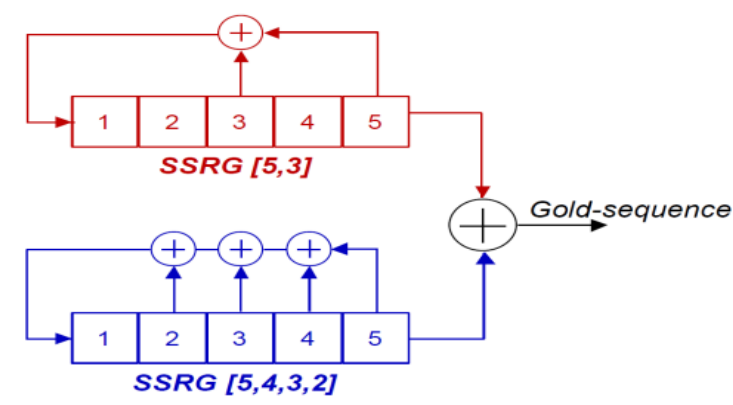

Figure.19 - Gold code generator $(\mathrm{PN} 1 \oplus \mathrm{PN} 2)$

Any 2-register Gold code generator of length $\mathrm{L}$ can generate $2 \mathrm{~L}-1$ sequence (length $2 \mathrm{~L}-1$ ) plus the two base $\mathrm{m}$-sequences, giving a total of $2 \mathrm{~L}+1$ sequences. In addition to their advantage in generating large numbers of codes, the Gold codes may be chosen so that over a set of codes available from a given generator the autocorrelation and the cross-correlation between the codes are uniform and bounded. When specially selected $\mathrm{m}$-sequences, called preferred m-sequences, are used the generated Gold codes have a three valued crosscorrelation.

\begin{tabular}{|c|c|c|c|c|c|c|}
\hline L & $N_{c}=2^{L}-1$ & preferred pairs of m-sequences & \multicolumn{3}{|c|}{$\begin{array}{c}\text { 3-value } \\
\text { crosscorrelations }\end{array}$} & bound \\
\hline 5 & 31 & {$[5,3][5,4,3,2]$} & 7 & -1 & -9 & $-29 \%$ \\
\hline 6 & 63 & {$[6,1][6,5,2,1]$} & 15 & -1 & -17 & $-27 \%$ \\
\hline 7 & 127 & $\begin{array}{l}{[7,3][7,3,2,1]} \\
{[7,3,2,1][7,5,4,3,2,1]}\end{array}$ & 15 & -1 & -17 & $-13 \%$ \\
\hline $8^{*}$ & 255 & {$[8,7,6,5,2,1][8,7,6,1]$} & 31 & -1 & -17 & $+12 \%$ \\
\hline 9 & 511 & $\begin{array}{l}{[9,4][9,6,4,3]} \\
{[9,6,4,3][9,8,4,1]}\end{array}$ & 31 & -1 & -33 & $-6 \%$ \\
\hline 10 & 1023 & $\begin{array}{l}{[10,9,8,7,6,5,4,3][10,9,7,6,4,1]} \\
{[10,8,7,6,5,4,3,1][10,9,7,6,4,1]} \\
{[10,8,5,1][10,7,6,4,2,1]}\end{array}$ & 63 & -1 & -65 & $-6 \%$ \\
\hline 11 & 2047 & $\begin{array}{l}{[11,2][11,8,5,2]} \\
{[11,8,5,2][11,10,3,2]}\end{array}$ & 63 & -1 & -65 & $-3 \%$ \\
\hline
\end{tabular}

Table.2 - The most important subset of preferred pair Gold codes [2] 


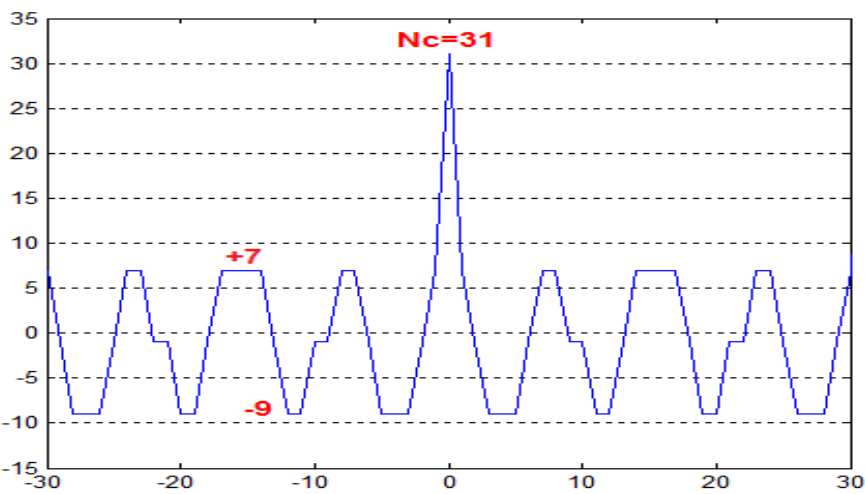

Figure.20 - Gold 1/Auto-Correlation

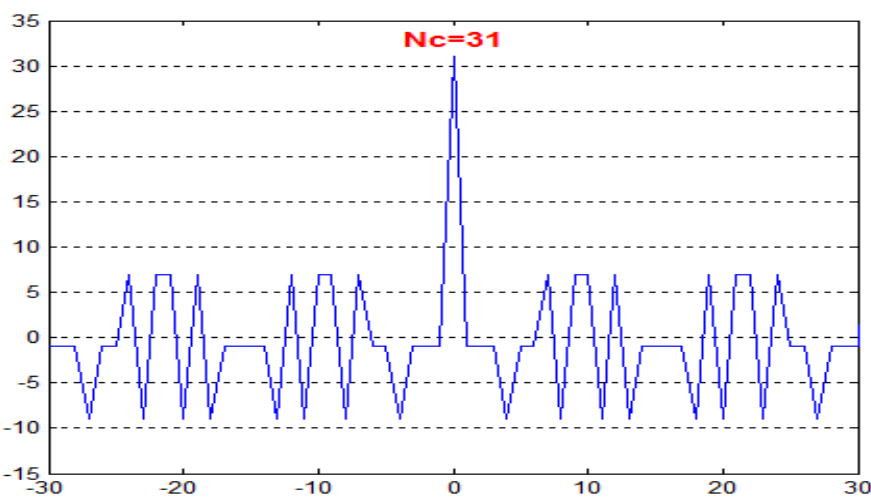

Figure.21 - Gold 2 / Auto-Correlation

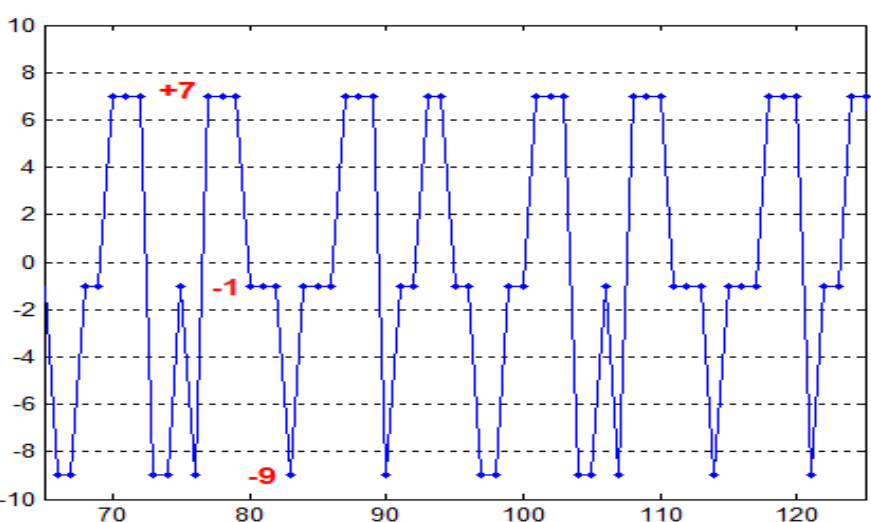

Figure.22 - Gold 1 \& Gold 2 / Cross-Correlation

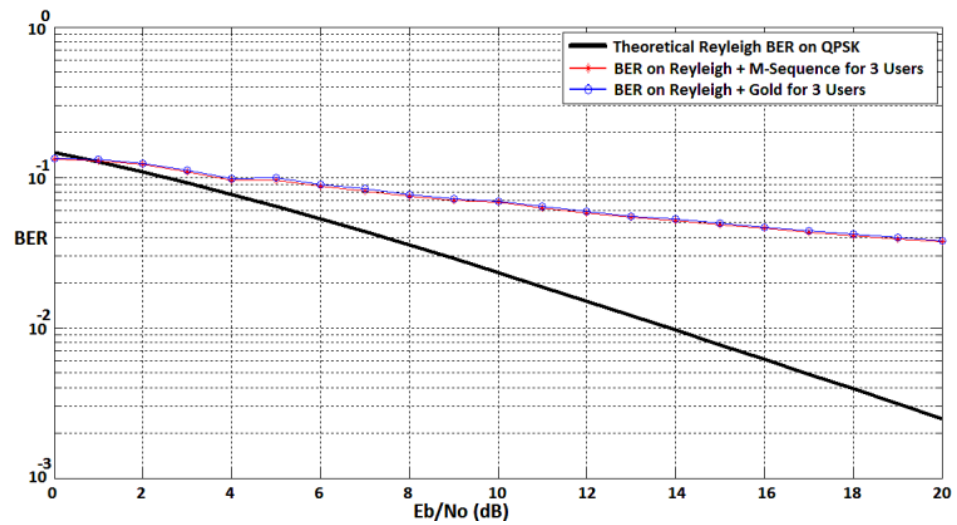

Figure.23 - DS-CDMA performance with M-Sequence and Gold codes in Reyleigh environment [7] 


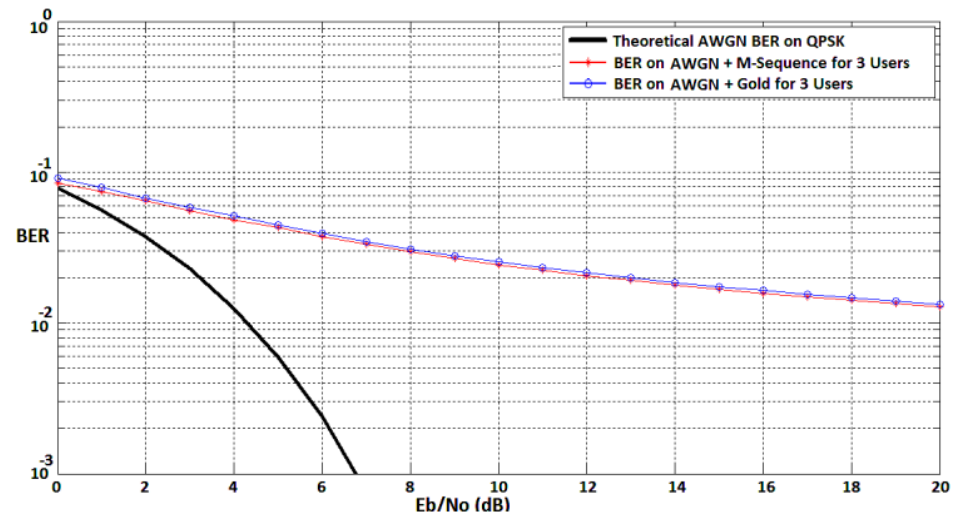

Figure.24 - DS-CDMA performance with M-Sequence and Gold codes in AWGN environment [7]

VIII. Conclusion

The performance measurement of two well-known and practical codes based on the autocorrelation, cross-correlation and BER values in tow fading channels are analyzed. The autocorrelation value for the both codes is high and proper for synchronization to lock the demodulator on the desire satellite or control terrestrial stations signal and also according to the obtained results of the BER values, both codes are almost identical behavior in Reyleigh and AWGN environments but it should be considered that to select an optimized code the cross-correlation value must be very low and shaped. This paper finds the spreading code with suitable autocorrelation properties along with low cross-correlation values between tow mentioned codes. The result expresses that Gold codes have a good autocorrelation properties which helps receivers to lock on the desire signal among the other signals and plus have the pairs with the low and shaped peak cross-correlation so that they can be more appropriate for using simultaneously several satellites telemetry data in a DS-CDMA systems.

\section{References}

[1]. CCSDS415.0-G-1. Green Book. Issue 1. Washington, D.C.: CCSDS, April 2013

[2]. J.G. Proakis,' Digital Communication, Prentice-Hall, 1994, chapter 12, 16

[3]. Viterbi, 'CDMA Principles of Spread Spectrum Communication', Addison-Wesley, 1995

[4]. R.C. Dixon, 'Spread Spectrum Systems with commercial applications', John Wiley \& Sons, Inc., 1994

[5]. Spectrum communications- A Tutorial, IEEE Trans. On Communications, vol. com-30, no. 5, May 1982 pp. 855 884

[6]. E.H. Dinan ve B. Jabbari, "Spreading codes for direct sequence CDMA and wideband CDMA cellular networks",

[7]. IEEE Communications Magazine, vol. 36, pp.48-54, Sep 1998

[8]. PERFORMANCE EVALUATION OF DS-CDMA SYSTEM USING MATLAB, International Journal of Advances in Engineering \& Technology, Jan 2012, ISSN: 2231-1963 